\title{
Clinical Application of the Quick Sepsis-Related Organ Failure Assessment Score at Intensive Care Unit Admission in Patients with Bacteremia: A Single-Center Experience of Korea
}

\author{
Hae Jung $\mathrm{Na}^{1}$, Eun Suk Jeong ${ }^{2}$, Insu Kim², Won-Young Kim², and Kwangha Lee ${ }^{2}$ \\ 'Department of Internal Medicine, Good Samsun Hospital, Busan; '2Division of Pulmonary, Allergy and Critical Care Medicine, Department of Internal Medicine, \\ Pusan National University School of Medicine, Busan, Korea
}

Background: We evaluated the clinical usefulness of the quick Sepsis-Related Organ Failure Assessment (qSOFA) score (based on the 2016 definition of sepsis) at intensive care unit admission in Korean patients with bacteremia.

Methods: We retrospectively analyzed clinical data from 236 patients between March 2011 and February 2016. In addition to the qSOFA, the Modified Early Warning score (MEWS) and systemic inflammatory response syndrome (SIRS) criteria were calculated.

Results: The patients' median age was 69 years, and $61.0 \%$ were male. Of the patients, $127(53.8 \%)$ had a qSOFA score $\geq 2$ points. They had significantly higher rates of septic shock, thrombocytopenia, and hyperlactatemia, and increased requirements for ventilator care, neuromuscular blocking agents, vasopressors, and hemodialysis within 72 hours after intensive care unit admission. They also had a significantly higher 28-day mortality rate. When analyzed using common thresholds (MEWS $\geq 5$ and $\geq 2$ SIRS criteria), patients with a MEWS $\geq 5$ had the same results as those with a qSOFA score $\geq 2(P<0.05)$. However, patients with $\geq 2$ SIRS criteria showed no significant differences.

Conclusions: Our results show that a qSOFA score $\geq 2$ at admission is a useful screening tool for predicting disease severity and medical resource usage within 72 hours after admission, and for predicting 28-day mortality rates in patients with bacteremia. In addition, qSOFA scores may be more useful than SIRS criteria in terms of prognostic utility.

Key Words: bacteremia; intensive care units; mortality; prognosis; sepsis.

\section{Introduction}

Sepsis is a life-threatening organ dysfunction resulting from a dysregulated host response to infection and is the leading cause of morbidity and mortality worldwide [1,2]. Sepsis was defined by a consensus conference in 1991 [3]; however, the definition was revised in 2016 (sepsis-3) [2]. In sepsis-3, the quick Sepsis-Related Organ Failure Assessment (qSOFA) score was introduced as a means of screening for sepsis at the bedside based on a patient's respiratory rate, blood pressure, and level of consciousness [2]. In addition, the recommendations suggest that patients with a qSOFA score $\geq 2$ suspected to have an infection should be monitored closely.

Although several studies have suggested that compliance with the Surviving Sepsis Campaign bundles can benefit sur-

Received on April 5, 2017 Revised on June 2, 2017 Accepted on July 20, 2017

Correspondence to: Kwangha Lee, Division of Pulmonary, Allergy and Critical Care Medicine, Department of Internal Medicine, Pusan National University School of Medicine, 179 Gudeok-ro, Seo-gu, Busan 49241, Korea

Tel: +82-51-240-7743, Fax: +82-51-245-3127,E-mail: jubilate@pusan.ac.kr

*No potential conflict of interest relevant to this article was reported.

(cc) This is an Open Access article distributed under the terms of the Creative Commons Attribution Non-Commercial License (http://creativecommons.org/ licenses/by-nc/4.0/) which permits unrestricted non-commercial use, distribution, and reproduction in any medium, provided the original work is properly cited. Copyright (c) 2017 The Korean Society of Critical Care Medicine 
vival [4-7], compliance with resuscitation and management bundles is generally poor in many Asian intensive care units (ICUs) (including Korea) [8,9]. Moreover, there have been no multicenter studies regarding the current status of compliance with management recommendations at the national level because critical care resources and facilities at university hospitals in Korea are limited compared with Western countries [10].

Therefore, it is questionable whether qSOFA scores can be applied successfully in Korea. In addition, no largescale multicenter studies have reported the prognostic utility of this score. Also, the usefulness of the qSOFA score in Korea is unknown.

We hypothesized the qSOFA score would be useful in Korean patients. The present study investigated the clinical application and usefulness of the qSOFA score at ICU admission for predicting 28-day mortality in patients with a microbiologically diagnosed infection. In addition, we compared this score with other conventional early warning scores $[3,11,12]$.

\section{Materials and Methods}

\section{1) Study design and subjects}

This retrospective study was conducted at a universityaffiliated tertiary care hospital. This hospital has six functionally separate ICUs with 85 beds (medical, 12 beds; surgical, 10 beds; cardio-stroke, 14 beds; neurosurgical, 13 beds; emergency, 20 beds; and trauma, 16 beds) with full cardiovascular and close airway monitoring. All patients were managed according to therapeutic recommendations based on Surviving Sepsis guidelines and a lung-protective ventilator strategy $[13,14]$.

We included patients who had various infectious causes with positive blood culture tests at ICU admission; all blood culture results were obtained within 3 days after ICU admission. The inclusion period was from March 2011 to February 2016. The exclusion criteria were patients younger than 18 years and those whose mental state could not be assessed. Also, patients who could not know 28-day mortality after ICU admission (example, transferred to other hospitals) were excluded. For all positive blood cultures, organism identification was performed by conventional and automated biochemical methods (VITEK 2; BioMérieux, Marcy l'Etoile, France) from March 2011 to February 2013, and matrix-assisted laser desorption/ionization time-of-flight mass spectrometry (Bruker Daltonic, Bremen, Germany) from March 2013 to February 2016. The medical records and laboratory and radiological findings of all patients included in the study were reviewed. All investigators confirmed that the study objectives and procedures were complete, and they had full access to all data. The investigators completed a case report form for each patient; data were collected from September to December in 2016. This study was conducted with the approval of Institutional Review Board of Pusan National University Hospital (IRB No. 1612-003-049). This study had no impact on patient treatment.

\section{2) Data collection}

The following data were gathered from the medical records of each patient: age, sex, comorbidities before ICU admission, ICU admission route, and length of stay (LOS; ICU and hospital). The severity of illness was measured by the Acute Physiology and Chronic Health Evaluation (APACHE) II score, and accompanying organ failure was measured by the Sequential Organ Failure Assessment (SOFA) score $[15,16]$. APACHE II and SOFA scores were calculated using data from the first 24 hours of ICU admission.

The qSOFA was calculated at the time of ICU admission, which was defined as a systolic blood pressure $\leq 100$ $\mathrm{mmHg}$, respiratory rate $\geq 22$ breaths per minute, and altered mental status (defined as a Glasgow Coma Scale score $\leq 13$ ) [2]. To compare the prognostic utility with qSOFA, we also calculated the Modified Early Warning score (MEWS) at ICU admission and systemic inflammatory response syndrome (SIRS) criteria within the first 24 hours of ICU admission; these data were based on previously published definitions $[3,11,12]$. 
Table 1. Comparison of baseline characteristics between survivors and non-survivors

\begin{tabular}{|c|c|c|c|c|}
\hline Characteristic & Total $(n=236)$ & Survivor $(n=139)$ & Non-survivor $(\mathrm{n}=97)$ & P-value \\
\hline Age (yr) & $69(57-76)$ & $70(58-77)$ & $67(55-75)$ & 0.192 \\
\hline Male sex & $144(61.0)$ & $82(59.0)$ & $62(63.9)$ & 0.498 \\
\hline \multicolumn{5}{|l|}{ ICU type } \\
\hline Medical ICU & $86(36.4)$ & $46(33.1)$ & $40(41.2)$ & 0.218 \\
\hline Surgical ICU & $31(31.1)$ & $14(10.1)$ & $17(17.5)$ & 0.117 \\
\hline Cardio-stroke ICU & $32(13.6)$ & $18(12.9)$ & $14(14.4)$ & 0.847 \\
\hline Emergency ICU & $57(24.2)$ & $13(28.1)$ & $18(18.6)$ & 0.122 \\
\hline Neurosurgical ICU & $27(11.4)$ & $19(13.7)$ & $8(8.2)$ & 0.219 \\
\hline Trauma ICU & $3(1.3)$ & $3(1.3)$ & 0 & 0.271 \\
\hline APACHE II score on ICU admission day & $23(17-29)$ & $20(15-26)$ & $26(22-32)$ & $<0.001$ \\
\hline SOFA score on ICU admission day & $7(4-9)$ & $5(3-8)$ & $9(6-11)$ & $<0.001$ \\
\hline qSOFA score at ICU admission time & $2(0-3)$ & $1(0-3)$ & $2(0-3)$ & $<0.001$ \\
\hline \multicolumn{5}{|l|}{ Comorbidities, overlapped } \\
\hline Diabetes mellitus & 69 (29.2) & $46(33.1)$ & $23(23.7)$ & 0.146 \\
\hline Hemato-oncological disease & $55(23.3)$ & $21(15.1)$ & $34(35.1)$ & 0.001 \\
\hline Cerebrovascular disease & $34(14.4)$ & $20(14.4)$ & $14(14.4)$ & $>0.999$ \\
\hline Heart failure & $34(14.4)$ & $21(15.1)$ & $13(13.4)$ & 0.851 \\
\hline Chronic kidney disease & 25 (10.6) & $14(10.1)$ & $11(11.3)$ & 0.831 \\
\hline Billary disease & $21(8.9)$ & $16(11.5)$ & $5(5.2)$ & 0.107 \\
\hline Chronic liver disease & $19(8.1)$ & $9(6.5)$ & $10(10.3)$ & 0.335 \\
\hline Neuromuscular disease & $13(5.5)$ & $11(7.9)$ & $2(2.1)$ & 0.079 \\
\hline Chronic lung disease $^{a}$ & $12(8.9)$ & $12(8.6)$ & $9(9.3)$ & $>0.999$ \\
\hline \multicolumn{5}{|l|}{ Source of infection } \\
\hline Pneumonia & $91(38.6)$ & 49 (35.3) & $42(43.3)$ & 0.224 \\
\hline Intra-abdominal & $61(25.8)$ & $37(26.6)$ & $24(24.7)$ & 0.765 \\
\hline Urinary tract & $25(10.6)$ & $19(13.7)$ & $6(6.2)$ & 0.085 \\
\hline Musculoskeletal & $41(17.4)$ & $28(20.1)$ & $13(13.4)$ & 0.222 \\
\hline Catheter-related & $22(9.3)$ & $14(10.1)$ & $8(8.2)$ & 0.821 \\
\hline Neutropenia & $13(5.5)$ & $2(1.4)$ & $11(11.3)$ & 0.002 \\
\hline Infectious endocarditis & $11(4.7)$ & $5(3.6)$ & $6(6.2)$ & 0.366 \\
\hline \multicolumn{5}{|l|}{ Organism } \\
\hline Gram-positive bacteremia & $138(58.5)$ & $92(66.2)$ & $46(47.4)$ & 0.005 \\
\hline Gram-negative bacteremia & $84(35.6)$ & $42(30.2)$ & $42(43.3)$ & 0.053 \\
\hline Multidrug-resistant bacteremia ${ }^{b}$ & $60(25.4)$ & $33(23.7)$ & $27(27.8)$ & 0.544 \\
\hline Fungemia & $24(10.2)$ & $10(7.2)$ & $14(14.4)$ & 0.082 \\
\hline Polymicrobial ${ }^{c}$ & 27 (11.4) & $14(10.1)$ & $13(13.4)$ & 0.534 \\
\hline
\end{tabular}

Values are presented as median (interquartile range) or number (\%).

ICU: intensive care unit; APACHE: Acute Physiology and Chronic Health Evaluation; SOFA: Sequential Organ Failure Assessment; qSOFA: quick Sepsis-Related Organ Failure Assessment.

${ }^{a}$ Chronic obstructive pulmonary disease, asthma, and bronchiectasis; ' Including methicillin-resistant Staphylococcus aureus, extended-spectrum B-lactamase-producing Gram-negative bacteria (Escherichia coli and Klebsiella pneumoniae), carbapenem-resistant Gram-negative rods (Acinetobacter baumannii and Pseudomonas aeruginosa), and vancomycin-resistant Enterococcus faecium; 'A blood culture test revealed more than two bacteria. 
We also evaluated primary sources of infection at ICU admission, microbiological data (Gram staining, organism identification, and susceptibility testing), and the requirement for hemodialysis (defined as the use of any form of renal replacement therapy), neuromuscular blocking agents, vasopressors, and ventilator care within 3 days after ICU admission. In addition, the blood platelet count and arterial lactic acid level were determined during the first 3 days after ICU admission. Survivors were defined as patients that survived for 28 days after ICU admission.

\section{3) Statistical analysis}

Continuous variables are expressed as medians (interquartile range [IQR]) and categorical variables are expressed as numbers (percentages). Student t-test and the Mann-Whitney U-test were applied to compare continuous variables. The chi-square and Fisher exact tests (for small numbers) were used to compare categorical variables. To estimate predictive capabilities of the qSOFA score and other scores for our cohort, the receiver operating characteristic curves were used to determine cutoff value. Pearson correlation coefficients between the qSOFA score and MEWS and SIRS were calculated. Logistic regression analyses were performed to evaluate the qSOFA score as an independent prognostic factor in 28day mortality. All statistical analyses were performed using SPSS version 19.0 (IBM Corp., Armonk, NY, USA). A two-tailed P-value $<0.05$ was considered to indicate significant difference.

\section{Results}

\section{1) Baseline characteristics}

During the study period, we identified 236 patients with infectious causes that had positive blood cultures within 3 days after ICU admission. In the total patient population, $151(64.0 \%)$ were admitted to the ICU via the emergency department (ED) and 144 patients (61.0\%) received ventilator care during their ICU stay. The me- dian ICU LOS and hospital LOS were 10 days (IQR, 5 to 19 days) and 25 days (IQR, 14 to 53 days), respectively. Diabetes mellitus was the most common underlying disease, and pneumonia was the most common source of bacteremia (Table 1). Gram-positive bacteria were the most commonly identified organisms (Table 1). Of total enrolled patients, 49 patients (20.8\%) received surgical drainage aside from antibiotics. The clinical characteristics of all patients enrolled in this study and comparisons between survivors and non-survivors are presented in Table 1

\section{2) qSOFA score and patient outcomes}

Figure 1 shows the number of patients for each qSOFA level and the corresponding mortality rates. Of the patients with a qSOFA score $\geq 2(\mathrm{n}=127), 38(29.9 \%)$ had all three criteria, followed by blood pressure and respiratory rate criteria $(\mathrm{n}=35,27.6 \%)$, mental status and blood pressure $(\mathrm{n}=28,22.0 \%)$, and mental status and respiratory rate criteria $(n=26,20.5 \%)$. Patients with a qSOFA score $\geq 2$ had significantly higher APACHE II and SOFA scores compared to those with a qSOFA score $<2$. In addition, these patients had significantly higher rates of septic shock, thrombocytopenia, and hyperlactatemia, and significantly greater requirements for ventilator care, neuromuscular blocking agents, vasopressors, and hemodialysis during the first 72 hours of ICU admission (Table 2). Fur-

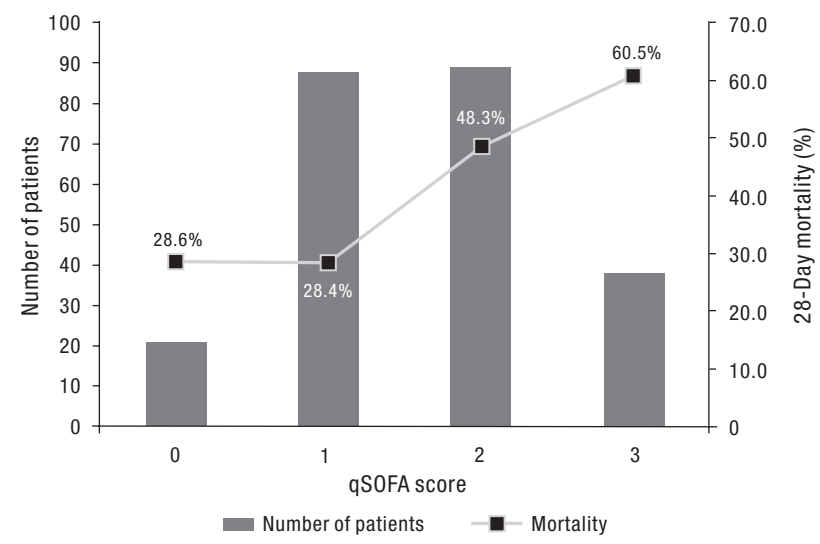

Figure 1. The number of patients for each qSOFA level (left $Y$ axis) and the corresponding mortality (right $Y$ axis). qSOFA: quick Sepsis-Related Organ Failure Assessment. 
Table 2. Comparison of clinical data from patients with a qSOFA $\geq 2$ or $<2$

\begin{tabular}{|c|c|c|c|}
\hline Variable & qSOFA $\geq 2(n=127)$ & qSOFA $<2(n=109)$ & P-value \\
\hline Age (yr) & $69(57-76)$ & $68(57-76)$ & 0.505 \\
\hline Male sex & 79 (62.2) & $65(59.6)$ & 0.691 \\
\hline APACHE II score & $25(19-31)$ & $21(15-26)$ & $<0.001$ \\
\hline SOFA score & $9(6-11)$ & $5(3-7)$ & $<0.001$ \\
\hline Hospital LOS (d) & $24(15-42)$ & $27(13-57)$ & 0.355 \\
\hline ICU LOS (d) & $11(5-19)$ & $9(4-18)$ & 0.540 \\
\hline \multicolumn{4}{|l|}{ Source of infection } \\
\hline Pneumonia & $58(45.7)$ & $33(30.3)$ & 0.016 \\
\hline Intra-abdominal & $36(28.3)$ & $25(22.9)$ & 0.373 \\
\hline Urinary tract & $12(9.4)$ & $13(11.9)$ & 0.672 \\
\hline Musculoskeletal & $17(13.4)$ & $24(22.0)$ & 0.088 \\
\hline Catheter-related & $11(8.7)$ & $11(10.1)$ & 0.823 \\
\hline Neutropenia & $11(8.7)$ & $2(1.8)$ & 0.024 \\
\hline Infective endocarditis & $4(3.1)$ & $7(6.4)$ & 0.354 \\
\hline \multicolumn{4}{|l|}{ Organism } \\
\hline Gram-positive bacteria & $64(50.4)$ & $74(67.9)$ & 0.008 \\
\hline Gram-negative bacteria & $56(44.1)$ & $28(25.7)$ & 0.004 \\
\hline Requirement for hemodialysis ${ }^{\mathrm{a}}$ & $55(43.3)$ & $29(26.6)$ & 0.009 \\
\hline Requirement for $\mathrm{NMBAs}^{\mathrm{a}}$ & $33(26.0)$ & $16(14.7)$ & 0.037 \\
\hline Requirement for vasopressors ${ }^{\mathrm{a}}$ & $93(73.2)$ & $53(48.6)$ & $<0.001$ \\
\hline Ventilator care ${ }^{\mathrm{a}}$ & $76(59.8)$ & $51(46.8)$ & 0.050 \\
\hline Thrombocytopenia ${ }^{\mathrm{a}, \mathrm{b}}$ & $102(80.3)$ & $62(56.9)$ & $<0.001$ \\
\hline Lactic acid >2.0 mmol/L $(n=161)^{\mathrm{a}}$ & $80(80.0)$ & $39(63.9)$ & 0.028 \\
\hline Septic shock $(n=161)^{a, c}$ & $67(67.0)$ & $29(47.5)$ & 0.020 \\
\hline 28-Day mortality & $66(52.0)$ & $31(28.4)$ & $<0.001$ \\
\hline
\end{tabular}

Values are presented as median (interquartile range) or number (\%).

qSOFA: quick Sepsis-Related Organ Failure Assessment; APACHE: Acute Physiology and Chronic Health Evaluation; SOFA: Sequential Organ Failure Assessment; LOS: length of stay; ICU: intensive care unit; NMBA: neuromuscular blocking agent.

${ }^{a}$ All clinical courses developed within 72 hours after ICU admission; ${ }^{b}$ Defined as a platelet count $\leq 150 \times 10^{9} / \mathrm{L}$; ${ }^{c}$ Based on the sepsis-3 consensus statement.

ther analysis indicated that patients with a qSOFA score $\geq 2$ had significantly higher 28-day mortality rates than those with a qSOFA score $<2$ (Table 2). A univariate logistic regression analysis showed that a qSOFA score $\geq 2$ was associated with 28-day mortality in our cohort (odds ratio, 2.722; $95 \%$ confidence interval, 1.582 to 4.683 ; $\mathrm{P}<0.001)$.

\section{3) Comparison of qSOFA score with MEWS, SIRS, and SOFA}

When we compared two conventional early warning scores (MEWS and SIRS), we found correlations be- tween qSOFA score and MEWS $(\gamma=0.401, \mathrm{P}<0.001)$ and between qSOFA score and SIRS criteria $(\gamma=0.271$, $\mathrm{P}<0.001)$. Also, positive correlation was found between qSOFA score and SOFA score $(\gamma=0.465, \mathrm{P}<0.001)$.

Further analysis using common thresholds for each conventional early warning score (MEWS $\geq 5$, qSO$F A \geq 2$, and SIRS criteria $\geq 2$ ) according to published data was presented in Table $3[3,11,17]$. Patients with a MEWS $\geq 5$ had significantly higher rates of septic shock, thrombocytopenia, and hyperlactatemia, and significantly greater requirements for ventilator care, neuromuscular blocking agents, vasopressors, and hemodialysis dur- 
Table 3. Comparison of clinical courses among the cutoff levels of some scores (MEWS $\geq 5$, SIRS criteria $\geq 2$, and SOFA score $\geq 7$ ) for 28-day mortality

\begin{tabular}{|c|c|c|c|c|c|c|c|c|c|}
\hline \multirow[b]{2}{*}{ Variable } & \multicolumn{3}{|c|}{ MEWS } & \multicolumn{3}{|c|}{ SIRS } & \multicolumn{3}{|c|}{ SOFA } \\
\hline & $\begin{array}{c}\geq 5 \\
(n=175)\end{array}$ & $\begin{array}{c}<5 \\
(n=61)\end{array}$ & P-value & $\begin{array}{c}\geq 2 \\
(n=228)\end{array}$ & $\begin{array}{c}<2 \\
(n=8)\end{array}$ & P-value & $\begin{array}{c}\geq 7 \\
(n=125)\end{array}$ & $\begin{array}{c}<7 \\
(n=111)\end{array}$ & P-value \\
\hline Requirement for hemodialysis ${ }^{\mathrm{a}}$ & $71(40.6)$ & $13(21.3)$ & 0.008 & $81(35.5)$ & $3(37.5)$ & $>0.999$ & $59(47.2)$ & $25(22.5)$ & $<0.001$ \\
\hline Requirement for NMBAs ${ }^{\mathrm{a}}$ & $45(25.7)$ & $4(6.6)$ & 0.001 & $49(21.5)$ & 0 & 0.211 & $35(28.0)$ & $14(12.6)$ & 0.004 \\
\hline Requirement for vasopressors ${ }^{\mathrm{a}}$ & $129(73.7)$ & $17(27.9)$ & $<0.001$ & $141(61.8)$ & $5(62.5)$ & $>0.999$ & $100(80.0)$ & $46(41.4)$ & $<0.001$ \\
\hline$V_{\text {entilator care }}{ }^{\mathrm{a}}$ & $113(64.6)$ & $14(23.0)$ & $<0.001$ & $123(53.9)$ & $4(50.0)$ & $>0.999$ & $81(64.8)$ & $46(41.4)$ & $<0.001$ \\
\hline Thrombocytopenia ${ }^{a, b}$ & $131(74.9)$ & $33(54.1)$ & 0.004 & $160(70.2)$ & $4(50.0)$ & 0.252 & $113(90.4)$ & $51(45.9)$ & $<0.001$ \\
\hline Lactic acid $>2.0 \mathrm{mmol} / \mathrm{L}(\mathrm{n}=161)^{\mathrm{a}}$ & $105(78.9)$ & $14(50.0)$ & 0.004 & $115(73.7)$ & $4(80.0)$ & $>0.999$ & $81(78.0)$ & $38(65.5)$ & 0.002 \\
\hline Septic shock $(n=161)^{a, c}$ & $84(65.4)$ & $9(32.1)$ & 0.001 & $92(59.0)$ & $4(80.0)$ & 0.649 & $72(64.9)$ & $24(25.0)$ & $<0.001$ \\
\hline 28-Day mortality & $84(48.0)$ & $13(21.3)$ & $<0.001$ & $134(58.8)$ & 5 (62.5) & $>0.999$ & 69 (55.2) & 28 (25.2) & $<0.001$ \\
\hline
\end{tabular}

Values are presented as number (\%). The cutoff levels of MEWS and SIRS were used according to published data, and the cutoff value of SOFA score was determined by receiver operating characteristic curves using our data.

MEWS: Modified Early Warning score; SIRS: systemic inflammatory response syndrome; SOFA: Sequential Organ Failure Assessment; NMBA: Neuromuscular blocking agent.

${ }^{a}$ All clinical courses were developed within 72 hours after ICU admission; 'Defined as a platelet count $\leq 150 \times 10^{9} / \mathrm{L}$; 'Based on the sepsis-3 consensus statement.

ing the initial 72 hours after ICU admission than those of MEWS $<5$ (Table 3). In addition, they had higher 28day mortality rates. However, patients with $\geq 2$ SIRS criteria showed no significant differences compared to those with $<2$ SIRS criteria (Table 3). Also, we found the cutoff value of SOFA was 7 , which were determined by receiver operating characteristic curves. When we compared between patients with a SOFA $\geq 7$ and $<7$, patients with a SOFA $\geq 7$ had same results as shown in patients with a MEWS $\geq 5$ (Table 3 ).

\section{Discussion}

In the present study, we enrolled patients with bacteremia on ICU admission and evaluated the clinical utility of qSOFA scores at the time of ICU admission. In the present study, qSOFA score had positive correlation with SOFA score. Also, a qSOFA score $\geq 2$ at ICU admission was associated with greater severity and higher medical resource use in the initial 72 hours after ICU admission. In addition, a qSOFA score $\geq 2$ was a significant prognostic indicator for 28-day mortality. Although critical care resources are typically limited and there are distinct cultural differences compared to those in Western countries $[9,10]$, our results suggested that a qSOFA score $\geq 2$ at ICU admission would be a useful screening tool for predicting disease severity and mortality in patients with bacteremia.

After introducing the qSOFA score in sepsis-3 as a screening tool for organ dysfunction [2], comparisons of qSOFA score with some conventional early warning scores were reported [17-20]. In our study, the cutoff levels of MEWS and SIRS were used according to previous reported data $[3,11,17]$. Our results showed a MEWS $\geq 5$ was associated with greater severity and higher medical resource use within 72 hours after ICU admission, and 28-day mortality after ICU admission, consistent with the observations associated with a qSOFA score $\geq 2$. However, we found no prognostic utility of $\geq 2$ SIRS criteria because $96.6 \%$ of the total patient population had $\geq 2$ SIRS criteria (Table 3 ). Our findings suggest that qSOFA scores may be more useful than SIRS criteria as a prognostic indicator, consistent with a previous report [21]. In comparison with previous studies [17-20], however, our patients had bacteremia with a documented infectious focus at ICU admission, and they had a higher mortality rate. Therefore, additional large-scale studies 
including patients with non-bacteremia are required to compare qSOFA with other early warning scores as early screening tools.

In the present study, we found the survival rate was different according to admission route. In patients admitted to ICU via ED, there was no significant difference in the 28-day mortality rate between patients with qSOFA score $\geq 2$ and $<2$ (37.5\% vs. $29.1 \%$, respectively; $\mathrm{P}=0.274)$. In patients admitted from general wards, however, patients with qSOFA score $\geq 2$ had significantly higher 28-day mortality rate than those with qSOFA score $<2$ ( $70.9 \%$ vs. $26.7 \%$, respectively; $\mathrm{P}<0.001)$. To find out these differences, we further evaluated where the patients were before being transferred to the emergency room of our hospital (i.e., home, heath care facility, or another teaching hospital), however, we could not investigate accurately because of the shortage of medical records. Therefore, further investigation is needed to evaluate the prognostic utility of qSOFA score for patients presenting to the ED.

Our study had several limitations. First, although qSOFA score was developed for patients with suspected infection presenting to the ED, in our study, we could not find the usefulness of qSOFA score for these patients due to the shortage of medical records. To assess the usefulness of this score, therefore, we enrolled patients who had documented infections with bacteremia admitted to ICU. Second, this study was conducted retrospectively; this may have resulted in information bias. Also, our enrolled patient populations were heterogeneous from six ICUs, which may be a bias. Third, our data represent the experience of a single center, so the results may not be representative of the general situation in Korea. Fourth, we expected that the qSOFA $\geq 2$ score was associated with poor prognosis according to documented bacteria or sources of infection; however, we were unable to identify statistical significances in subgroup analysis due to the small sample size.

In conclusion, we investigated the prognostic utility of the qSOFA score at ICU admission for patients with bacteremia. Our results show that a qSOFA score $\geq 2$ at admission could be useful as a screening tool for predicting clinical severity and medical resource use within 72 hours after admission, and for predicting the 28-day mortality rate. In addition, a comparison of qSOFA score with MEWS and SIRS criteria suggested that qSOFA scores are more useful than SIRS criteria. Prospective and large-scale studies are required to determine the prognostic utility of qSOFA scores in Korean ICUs.

\section{Acknowledgments}

This work was supported by the National Research Foundation of Korea (NRF) grant funded by the Korea government (Ministry of Science and ICT) (No. 2016R1C1B1008529).

\section{ORCID}

Won-Young Kim http://orcid.org/ 0000-0002-6038-9818

Kwangha Lee http://orcid.org/0000-0001-9878-201X

\section{References}

1. Fleischmann C, Scherag A, Adhikari NK, Hartog CS, Tsaganos T, Schlattmann P, et al. Assessment of global incidence and mortality of hospital-treated sepsis: current estimates and limitations. Am J Respir Crit Care Med 2016;193:259-72.

2. Singer M, Deutschman CS, Seymour CW, ShankarHari M, Annane D, Bauer M, et al. The third international consensus definitions for sepsis and septic shock (sepsis-3). JAMA 2016;315:801-10.

3. Bone RC, Balk RA, Cerra FB, Dellinger RP, Fein AM, Knaus WA, et al. Definitions for sepsis and organ failure and guidelines for the use of innovative therapies in sepsis: the ACCP/SCCM Consensus Conference Committee. American College of Chest Physicians/Society of Critical Care Medicine. Chest 1992;101:1644-55.

4. Levy MM, Rhodes A, Phillips GS, Townsend SR, 
Schorr CA, Beale R, et al. Surviving Sepsis Campaign: association between performance metrics and outcomes in a 7.5-year study. Crit Care Med 2015;43:3-12.

5. Rhodes A, Phillips G, Beale R, Cecconi M, Chiche JD, De Backer D, et al. The Surviving Sepsis Campaign bundles and outcome: results from the International Multicentre Prevalence study on Sepsis (the IMPreSS study). Intensive Care Med 2015;41:1620-8.

6. Herrán-Monge R, Muriel-Bombín A, GarcíaGarcía MM, Merino-García PA, Cítores-González R, Fernández-Ratero JA, et al. Mortality reduction and long-term compliance with surviving sepsis campaign: a nationwide multicenter study. Shock 2016;45:598-606.

7. Damiani E, Donati A, Serafini G, Rinaldi L, Adrario E, Pelaia P, et al. Effect of performance improvement programs on compliance with sepsis bundles and mortality: a systematic review and meta-analysis of observational studies. PLoS One 2015;10:e0125827.

8. Phua J, Koh Y, Du B, Tang YQ, Divatia JV, Tan CC, et al. Management of severe sepsis in patients admitted to Asian intensive care units: prospective cohort study. BMJ 2011;342:d3245.

9. Kim JH, Hong SK, Kim KC, Lee MG, Lee KM, Jung SS, et al. Influence of full-time intensivist and the nurse-to-patient ratio on the implementation of severe sepsis bundles in Korean intensive care units. J Crit Care 2012;27:414.e11-21.

10. Kwak SH, Jeong CW, Lee SH, Lee HJ, Koh Y. Current status of intensive care units registered as critical care subspecialty training hospitals in Korea. J Korean Med Sci 2014;29:431-7.

11. Subbe CP, Kruger M, Rutherford P, Gemmel L. Validation of a modified Early Warning Score in medical admissions. QJM 2001;94:521-6.

12. Levy MM, Fink MP, Marshall JC, Abraham E, Angus D, Cook D, et al. $2001 \mathrm{SCCM} / \mathrm{ESICM} / \mathrm{ACCP} /$ ATS/SIS International Sepsis Definitions Conference. Crit Care Med 2003;31:1250-6.

13. Dellinger RP, Levy MM, Rhodes A, Annane D, Ger- lach H, Opal SM, et al. Surviving Sepsis Campaign: international guidelines for management of severe sepsis and septic shock, 2012. Intensive Care Med 2013;39:165-228.

14. Acute Respiratory Distress Syndrome Network, Brower RG, Matthay MA, Morris A, Schoenfeld D, Thompson BT, et al. Ventilation with lower tidal volumes as compared with traditional tidal volumes for acute lung injury and the acute respiratory distress syndrome. N Engl J Med 2000;342:1301-8.

15. Knaus WA, Draper EA, Wagner DP, Zimmerman JE. APACHE II: a severity of disease classification system. Crit Care Med 1985;13:818-29.

16. Vincent JL, Moreno R, Takala J, Willatts S, De Mendonça A, Bruining H, et al. The SOFA (Sepsis-related Organ Failure Assessment) score to describe organ dysfunction/failure: on behalf of the Working Group on Sepsis-Related Problems of the European Society of Intensive Care Medicine. Intensive Care Med 1996;22:707-10.

17. Churpek MM, Snyder A, Han X, Sokol S, Pettit N, Howell MD, et al. Quick Sepsis-Related Organ Failure Assessment, systemic inflammatory response syndrome, and Early Warning Scores for detecting clinical deterioration in infected patients outside the intensive care unit. Am J Respir Crit Care Med 2017;195:906-11.

18. Williams JM, Greenslade JH, McKenzie JV, Chu K, Brown AFT, Lipman J. Systemic inflammatory response syndrome, quick sequential organ function assessment, and organ dysfunction: insights from a prospective database of ED patients with infection. Chest 2017;151:586-96.

19. Freund Y, Lemachatti N, Krastinova E, Van Laer M, Claessens YE, Avondo A, et al. Prognostic accuracy of sepsis-3 criteria for in-hospital mortality among patients with suspected infection presenting to the emergency department. JAMA 2017;317:301-8.

20. Raith EP, Udy AA, Bailey M, McGloughlin S, MacIsaac C, Bellomo R, et al. Prognostic accuracy of the SOFA score, SIRS criteria, and qSOFA score for 
in-hospital mortality among adults with suspected infection admitted to the intensive care unit. JAMA 2017;317:290-300.

21. Seymour CW, Liu VX, Iwashyna TJ, Brunkhorst
FM, Rea TD, Scherag A, et al. Assessment of clinical criteria for sepsis: for the third international consensus definitions for sepsis and septic shock (sepsis-3). JAMA 2016;315:762-74. 